\title{
A novel laparoscopic surgical device design in order to achive easy encircling and hanging manuevers in laparoscopic surgery
}

\author{
Tufan Egeli
}

Department of General Surgery, Division of Hepatopancreatobiliary Surgery, Dokuz Eylul University, Izmir, Turkey

Dear editor,

Encircling the vessels, tissues or some organs is one of the crucial maneuvers in all kind of abdominal surgeries. Because clamping, clipping or cutting the structures during surgery can only be performed by encircling and hanging them via a surgical tape. Pringle Maneuver which is used in hepatobiliary surgery is one of the most typical samples for it. ${ }^{[1]}$ In open surgical procedures, encircling maneuver can be performed with various angled or curved surgical tools such as right angle dissector, as well as the effective use of hands, wrists and arms. However, narrow port entry openings in laparoscopic surgery limit the usage of angled instruments. Thus, encircling maneuver is more difficult to perform when compared to open procedures.

Although there are various angled or curved tools designed for laparoscopic surgery, these instruments may be insufficient in some cases. ${ }^{[1]}$ Thus, the need for a laparoscopic surgical instrument, which will make easier and effective encircling continues. To meet this necessity, we designed a novel laparoscopic surgical instrument.

The instrument is inert and consists of two parts. The first part (inner part) passes through the second (outer part) (Fig. 1). Body of the inner part is rigid, can be made of plastic or metal. Tip of the inner part is made of 180

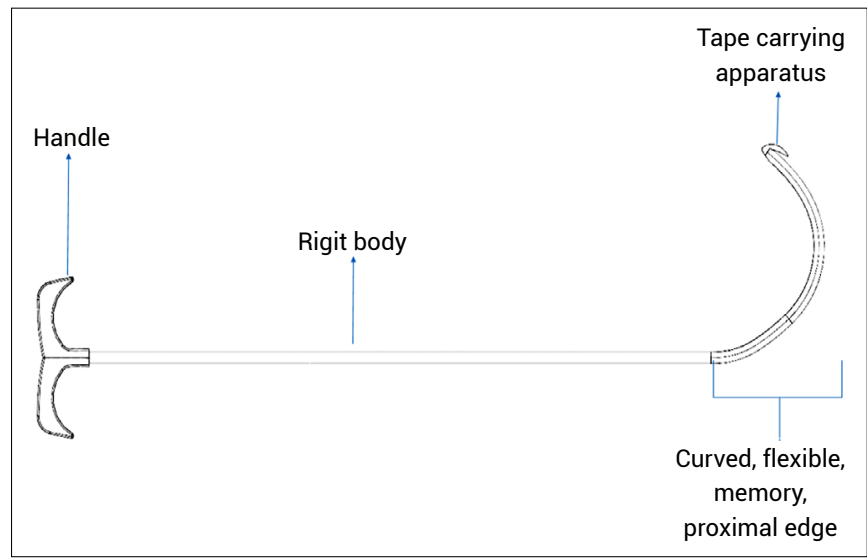

Figure 1. First (inner) part and its sections.

degrees curved braided steel wire which has elastic and flexible structure and may take shape against the force applied without breaking, and it can return to its original form thanks to its memory when the force disappears. $[2,3]$ The instrument's working principle is based on these properties of the tip of inner part. Tip of the inner part also has a flexable small apparatus which is designed to carry the surgical tape. By means of this tape carrying mechanism, catching the tape after encircling the structure and hanging maneuver would be faster and easier.

The outer part is a rigid tubular cover that made of plastic or metal and inner part passes through inside it. This tu- 
buler shaped outer part has a valve system at the entrance of it to prevent intaabdominal gas leak. In addition, the proximal proportion of outer part is designed slightly curved in order to see the inner part during movement and to aid blunt dissections.

The manuever is started by straightening the tip of the inner part, then advancing it through the second part. The tip of the inner part which is originally semicircular becomes straight thanks to its flexible structure and it keeps this straight shape during its advance in the outer part. When we push the inner part from the hole that placed at the end of the outer part, it starts to turn its original semicircular curved form due to its elastic and memory nature and allows to encirle the structures (Fig. 2). In addition, this device can provide us easier manuevers to hang the structures, if a surgical tape is attached to carrying apparatus which is placed at the end of the inner part. This device is designed as disposible, but reusable alternatives may be developed in time just like the other laparoscopic devices.

Compared to the other articulated devices that were developed to aim laparoscopic encircling maneuvers, our new device does not need extra area to perform these manuevers. Because it immediately starts to turn around the structures owing to its elastic, curved form with memory nature and can be used even in narrow places. Thus, this device may meet the necessaty of a comfortable encircling and hanging manouver in laparoscopic surgeries. Patent application for this device was done to Turk Patent in 10.10.2018

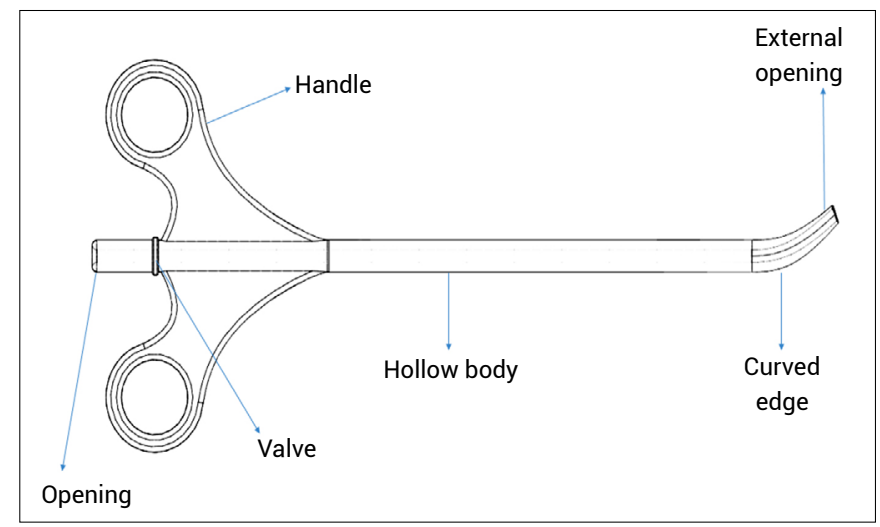

Figure 2. Second (outer) part and its sections.

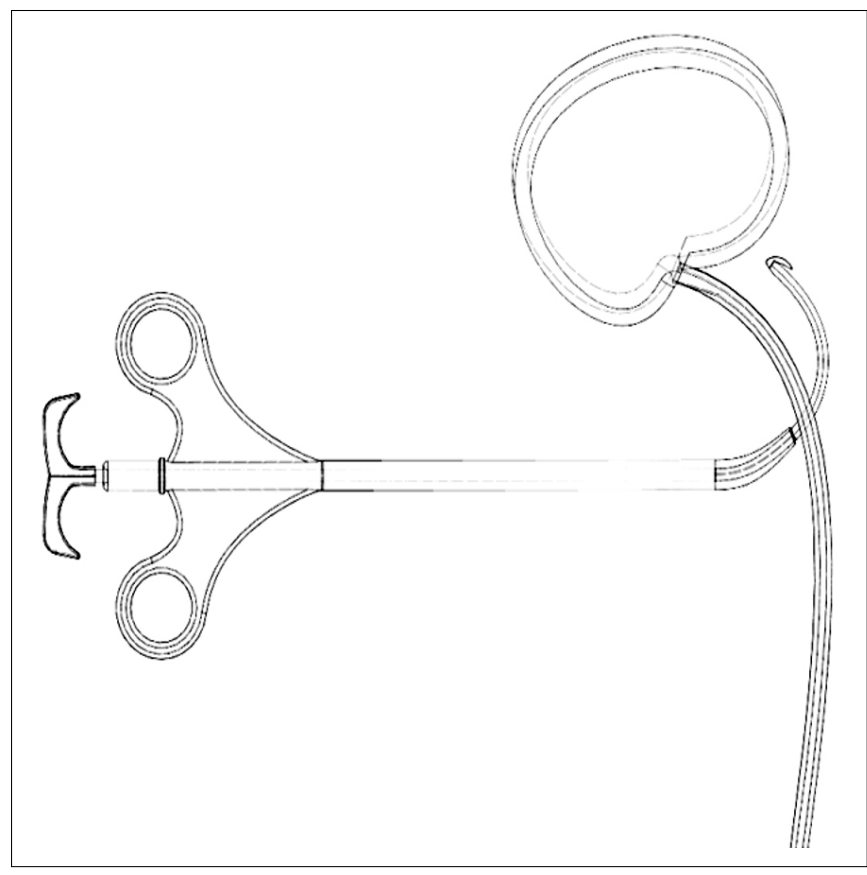

Figure 3. Viev of two parts union configuration. Encircling of the vessel or organ is completed. If surgical tape is attached the flexible metalic apparatus that placed at the tip, tape can be easily caught from other side and hanging manuever can be done.

and consideration is still ongoing. Also we will produce the first prototype of this device in short time with support of Dokuz Eylul University. Developments regarding this new laparoscopic tool will be shared with the national and the international surgical communities. Special thanks to Batu Manoglu for his valuable contributions.

\section{Disclosures}

Peer-review: Externally peer-reviewed.

Conflict of Interest: None declared.

\section{References}

1. Piardi $T$, Lhuaire $M$, Memeo $R$, Pessaux $P$, Kianmanesh $R$, Sommacale D. Laparoscopic Pringle maneuver: how we do it? Hepatobiliary Surg Nutr 2016;5:345-9. [CrossRef]

2. Pringle JH. V. Notes on the Arrest of Hepatic Hemorrhage Due to Trauma. Ann Surg 1908;48:541-9. [CrossRef]

3. Kusy RP, Dilley GJ. Elastic property ratios of a triple-stranded stainless steel arch wire. Am J Orthod 1984;86:177-88. 